\title{
Correlación entre Pobreza Extrema y Discapacidad en los Departamentos de Colombia
}

\section{Correlation between Extreme Poverty and Disability in the Departments of Colombia}

\author{
Yaneth Herazo Beltrán ${ }^{1}$ \\ Universidad Simón Bolívar \\ Regina Domínguez Anaya ${ }^{2}$ \\ Universidad San Buenaventura
}

\section{RESUMEN}

Introducción: Aparte de ser un problema médico, la discapacidad es un fenómeno socioeconómico, ya que una persona en situación de discapacidad tiene mayores restricciones para acceder, por ejemplo, a un empleo. Objetivo: Se propone estimar la correlación entre pobreza extrema y discapacidad en los departamentos de Colombia, según los datos del Censo General de 2005. Materiales y Métodos: Se realizó un estudio analítico ecológico. Como indicadores de discapacidad, se tomaron los datos del Departamento Administrativo Nacional de Estadísticas (DANE) y el indicador pobreza extrema del Departamento Nacional de Planeación (DNP). Se determinó el coeficiente de correlación (r) mediante el método de regresión lineal, usando el programa SPSS. Resultados: El análisis estableció la relación positiva entre las variables estudiadas, así: entre limitaciones permanentes para caminar y pobreza extrema, la correlación fue más fuerte $(\mathrm{r}=0,227)$, pero no significativamente estadística $(\mathrm{p}=0,286)$; igual, entre pobreza extrema y las limitaciones para autocuidado $(\mathrm{r}=0,161)$ y para el uso de brazos y manos $(\mathrm{r}=0,139)$, fue positiva, aunque débil. Conclusión: Los anteriores coeficientes de correlación (r) indican que la pobreza influye en la situación de discapacidad de las personas en forma acorde con la actividad que se analice, lo cual permite concluir que la pobreza es un factor contextual influyente en la discapacidad.

Palabras Clave: discapacidad, pobreza extrema, limitaciones funcionales (Fuente: DeCS).

\begin{abstract}
Introduction: Disability is considered a socio-economic phenomenon; a person in situation of disability has greater restrictions to access to employment. Objective: To estimate the correlation if any exists, between extreme poverty and disability in Colombian regions. The study is to be based on the 2005 general census. Materials and Methods: Analytic and ecologic study. 2005 general census data from the National Beauro of Statistical Administration (DANE) was used to form indicators of disability. For extreme poverty, indicators from the National Planning Bearue (DNP) were collected. The correlation coefficient (r) was determined through the linear regression method utilizing the SPSS software application. Results: The analysis establishes a positive correlation between permanent walking limitations and extreme poverty $(\mathrm{r}=0,227)$; however, not significant $(\mathrm{p}=0,286)$. Likewise, a positive correlation was found between extreme poverty and self-care $(r=0,161)$; between extreme poverty and use of arms and legs ( $r=0,139)$. The correlation is weaker for the latter parameters. Conclusion: The obtained correlation coefficients (r) indicate that poverty affects the disability condition based on the analyzed activity. Further, the results lead us to conclude that poverty is a contextual factor around disability. It is recommended that other analytical methods be utilized to establish causal relationships between disability and poverty at an individual level. The purpose would be to obtain a deeper understanding of how personal and environmental conditions lead to the social exclusion of individuals with disabilities.
\end{abstract}

Keywords: Disability, extreme poverty, functional limitations (Source: MeSH, NLM).

Fisioterapeuta. MSc Salud Pública. Correo electrónico: aherazo4@unisimonbolivar.edu.co

Fisioterapeuta. Especialista en Epidemiología. Correo electrónico: reginado90@ hotmail.com 


\section{Introducción}

La Clasificación Internacional del Funcionamiento de la Discapacidad y de la Salud (CIF) define la discapacidad como un término genérico, que incluye déficit, deficiencias o alteraciones en las funciones y/o estructuras corporales, limitaciones en la actividad y restricciones en la participación. También indica los aspectos negativos de la interrelación entre un individuo (con una condición de salud particular) y sus factores contextuales, los cuales constituyen en conjunto el contexto completo de la vida de un individuo, y en concreto el trasfondo sobre el que se clasifican los estados de salud en la CIF (1).

Los dos componentes de los factores contextuales son, a su vez, los factores ambientales y los factores personales. Los primeros se refieren a todos los aspectos del mundo extrínseco o externo que forma el contexto de la vida de un individuo, y como tal afecta el funcionamiento de esa persona; incluye tanto el mundo físico natural como el mundo físico creado por los seres humanos, las actitudes sociales, las características arquitectónicas, el clima, la geografía, y las estructuras legales y sociales (2). Los factores personales son todos los relacionados con el individuo, como edad, sexo, nivel social, experiencias vitales, etc.

Con la anterior caracterización, se reconoce, entonces, la multiplicidad de factores que conllevan a una situación de discapacidad. Debido a la multicausalidad de la discapacidad y a los efectos que tienen sobre esta situación los diversos factores, es importante estimar su interrelación para demostrar la fuerza de asociación entre las limitaciones funcionales de un individuo y dichos factores (3).

Uno de los factores contextuales es la pobreza extrema. De acuerdo con el CONPES (4), el índice de pobreza extrema no es otra cosa que el porcentaje de la población que percibe ingresos inferiores al valor de una canasta normativa de alimentos. Entonces, si la discapacidad es considerada un fenómeno socioeconómico, una persona en situación de discapacidad tiene limitada la capacidad para trabajar $\mathrm{y}$, en consecuencia, mayor probabilidad de pérdida laboral, disminución de ingresos, bajos índices de satisfacción y bienestar, y un menor consumo de bienes y servicios (5).
Mitra (6) expresa que la discapacidad es el lado oculto de la pobreza africana, y que durante muchos años ha existido un "círculo vicioso" cuyos componentes son la pobreza y la discapacidad, pues, en muchos casos, la situación de discapacidad en un individuo impide que se desempeñe en un empleo. Esta situación genera un incremento de los costos, por ejemplo, los costos relacionados con la salud, y dificulta el cubrimiento de ellos por parte de la familia. Por otra parte, la pobreza puede privar a las familias de sus recursos básicos y crear así condiciones a partir de las cuales surge la discapacidad (5).

Como puede verse, la probabilidad de que las personas en situación de discapacidad experimenten condiciones de pobreza es mayor que para aquellas que no presentan esta situación de salud (7). Además, la mayoría de las personas en situación de discapacidad ven afectadas sus oportunidades de asistir a la escuela, trabajar o vivir, disfrutar una vida familiar y participar como iguales en la vida social. Se estima, por último, que sólo $2 \%$ de personas en situación de discapacidad en los países en desarrollo tiene acceso a rehabilitación y a servicios básicos apropiados (8).

Por otra parte, existe una alta prevalencia de limitaciones funcionales en los adultos mayores que viven en áreas socioeconómicamente desfavorecidas, evidenciándose la importancia de direccionar las políticas de salud pública y la asignación de recursos a dichas poblaciones (9).

En Colombia, no es suficientemente clara la forma cómo el ambiente físico y social propician y exacerban la discapacidad, variables éstas que permiten una aproximación a la inclusión y participación de esta población; pero, según Cruz (10), existe una relación entre pobreza y discapacidad. Este autor precisa que los hogares con personas en situación de discapacidad tienden a tener menores ingresos por absorción laboral, dependencia económica, bajo nivel de estudio y restricciones para acceder al empleo.

Lo que sí resulta claro es que cuando se mejora el acceso a las fuentes de trabajo de la población en edad laboral, por ejemplo, cuando se hacen esfuerzos sostenibles para mejorar la educación y las adaptaciones en los puestos laborales, se reducen los índices de pobreza entre las personas en situación de discapacidad (6). 
Precisamente, en cuanto a la pobreza, el gobierno colombiano, en colaboración con el Banco Mundial y otros organismos multilaterales, ha determinado que las personas en situaciones de pobreza se encuentran más expuestas al riesgo. Los pobres tienen un menor acceso a los medios que permiten un manejo efectivo del riesgo, lo cual hace que su situación se agudice con el tiempo (12).

Como se ha dicho, a pesar de la obvia interrelación entre discapacidad y pobreza, en Colombia, es poca la información estadística confiable sobre esta premisa (7). Los investigadores desconocen la existencia de un estudio ecológico, que correlacione a nivel grupal y departamental los indicadores de pobreza extrema y discapacidad a partir de los datos del Censo general del año 2005 realizado por el DANE (11). Por lo tanto, correlacionar la pobreza extrema y la discapacidad, desde una perspectiva poblacional, permitiría estimar el porcentaje de influencia de la pobreza sobre la discapacidad y, a su vez, generar hipótesis acerca de la probable relación entre estas dos variables.

Por demás, la presente investigación toma como referente el enfoque de las limitaciones en la actividad utilizado en el Censo General de 2005, para recoger información sobre discapacidad. En este caso, se entiende por limitación en la actividad a las dificultades que tiene una persona para realizarla. Ello abarca desde una desviación leve hasta una grave, en términos de calidad y cantidad (11). De las limitaciones y dificultades en la realización de actividades y participación contempladas en el Censo General de 2005, se eligieron como indicadores de discapacidad: limitaciones permanentes para caminar o moverse, limitaciones permanentes para usar brazos y manos y limitaciones permanentes para el autocuidado. En este orden de ideas, la investigación tuvo como objetivo estimar la correlación existente entre la pobreza extrema y la discapacidad en los departamentos de Colombia, según los datos del Censo General 2005.

\section{Materiales y Métodos}

Esta es una investigación de tipo ecológico, analítica y retrospectiva. La unidad de observación la constituyen grupos de personas que viven en diferentes áreas geográficas, los departamentos de Colombia, y los datos han sido obtenidos con otros objetivos y no con los fines de este estudio. A partir de los datos recopilados en el Censo realizado por el Departamento Administrativo Nacional de Estadísticas (DANE) en el año 2005 y los del Departamento Nacional de Planeación (DNP), se estima la correlación existente entre las dos variables de estudio, la discapacidad y la pobreza extrema, mediante el método de regresión lineal.

La presente investigación compara el porcentaje de limitaciones permanentes para caminar o moverse, limitaciones permanentes para usar brazos y manos y limitaciones permanentes para el autocuidado, con el porcentaje de pobreza extrema en cada departamento colombiano durante el mismo período: el año 2005.

En los estudios de tipo ecológico, se busca tamizar hipótesis de asociación mediante datos poblacionales, y los resultados se estiman sobre grupos poblacionales, nunca sobre individuos. Estos estudios permiten plantear futuros análisis más complejos, que conlleven a inferencias causales entre estas dos variables. Inicialmente, se recopilaron los datos del Departamento Administrativo Nacional de Estadísticas (DANE) en la dirección electrónica www.dane.gov.co; y del Departamento Nacional de Planeación (DNP) en www.dnp.gog.co.

Los datos obtenidos de las diferentes fuentes de datos se analizaronmediante modelos lineales de regresión. En tanto que los porcentajes de discapacidad por departamento se compararon con los porcentajes de pobreza extrema, mediante el análisis de correlación, que expresa cómo cambian conjuntamente las variables de estudio.

El parámetro que refleja la correlación entre las dos variables se conoce como coeficiente de correlación (r). Este parámetro estima la fuerza de la asociación entre estas dos variables continuas. Del presente análisis, se pudo obtener en concreto un coeficiente de correlación positivo, el cual indica que al aumentar el porcentaje de pobreza, aumenta el porcentaje de discapacidad en un departamento; o un coeficiente de correlación negativo, indicando que si el valor de pobreza extrema aumenta, el porcentaje de discapacidad disminuye.

Los datos se organizaron según el método de regresión lineal para asociaciones bivariantes. Se utilizó el programa SPSS, de manera que en el eje de las Y se colocó la variable discapacidad y en el eje de la $\mathrm{X}$, la variable pobreza extrema. 


\section{Resultados}

Como se observa en la Tabla 1 , en los 24 departamentos de Colombia estudiados, se observan diversas fracciones de población en condición de pobreza extrema y con limitaciones permanentes para caminar, para su autocuidado y para usar brazos y manos.

Tabla 1. Proporción de pobreza extrema y limitaciones para autocuidado, caminar y usar brazos y manos, según datos del DANE y DNP (2005)

\begin{tabular}{lcccc}
\hline DEPARTAMENTO & $\begin{array}{c}\text { PROBREZA } \\
\text { EXTREMA \% }\end{array}$ & $\begin{array}{c}\text { LIMITACIONES PARA } \\
\text { AUTOCUIDADO \% }\end{array}$ & $\begin{array}{c}\text { LIMITACIONES } \\
\text { PARA CAMINAR \% }\end{array}$ & $\begin{array}{c}\text { LIMITACIONES } \\
\text { PARA USAR } \\
\text { BRAZOS Y } \\
\text { PIERNAS \% }\end{array}$ \\
\hline Antioquia & 18,5 & 9.6 & 31 & 15.7 \\
Atlántico & 12 & 14.6 & 25,9 & 15.5 \\
Bogotá & 4 & 10.2 & 26 & 14.7 \\
Bolívar & 13 & 12.4 & 27,7 & 13.8 \\
Boyacá & 35 & 7.2 & 36,7 & 15.6 \\
Caldas & 15,5 & 8.2 & 30,7 & 14.7 \\
Caquetá & 26 & 6.5 & 27,5 & 13.8 \\
Cauca & 27,5 & 6 & 28,1 & 14.7 \\
Cesar & 15 & 13.4 & 29,1 & 16.3 \\
Choco & 48 & 9.5 & 27,8 & 13.7 \\
Córdoba & 28 & 12.6 & 28,3 & 14.3 \\
Cundinamarca & 16 & 8.5 & 32,5 & 15.1 \\
Guajira & 16 & 14.9 & 26,8 & 13.8 \\
Huila & 24,5 & 7.3 & 30 & 15.5 \\
Magdalena & 15,5 & 13.5 & 27,8 & 16.2 \\
Meta & 12,5 & 10.3 & 35,5 & 15.9 \\
Nariño & 24 & 5.8 & 31,8 & 14.1 \\
Norte Santander & 18 & 8.3 & 28,7 & 13.2 \\
Quindío & 12,5 & 7 & 27 & 13 \\
Risaralda & 11 & 7.9 & 27,8 & 13.8 \\
Santander & 14,5 & 8.5 & 32,1 & 14.3 \\
Sucre & 23 & 12.8 & 28,6 & 12.9 \\
Tolima & 20 & 8.1 & 31,2 & 14.6 \\
Valle & 10 & 9 & 26,5 & 13.8 \\
\hline Fuente: elaborado por las autoras & conse en los resultados del Censo General de 2005 y lo datos del Departamento Nacional de Planeación.
\end{tabular}

Los resultados del modelo lineal de regresión indican que la pobreza tiene un importante efecto sobre las actividades de la vida diaria como caminar, el autocuidado y el uso de brazos y manos en los departamentos más pobres de Colombia. Se observa que existe una relación positiva entre las variables estudiadas, preciándose que la correlación fue más fuerte $(r=0,227)$ entre las limitaciones para caminar y pobreza extrema, pero no significativamente estadística $(\mathrm{p}=0,286)$ (Figura 1). Por otro lado, se evidencia que la correlación es positiva, aunque débil, entre la pobreza extrema, las limitaciones para las actividades de autocuidado ( $\mathrm{r}=0,161)$ (Figura 2) y las limitaciones para las actividades donde el sujeto usa brazos y manos $(\mathrm{r}=0,139)$ (Figura 3 ).

Las Figuras 1, 2 y 3 muestran, de otro lado, una gran dispersión entre los puntos que representan a los departamentos de Colombia, lo cual demuestra las diferencias en los niveles de pobreza y limitaciones para caminar, de autocuidado y para el uso de brazos y manos entre regiones. Esto significa que en Colombia existe una variación en la distribución de la pobreza y, por tanto, de la discapacidad. 


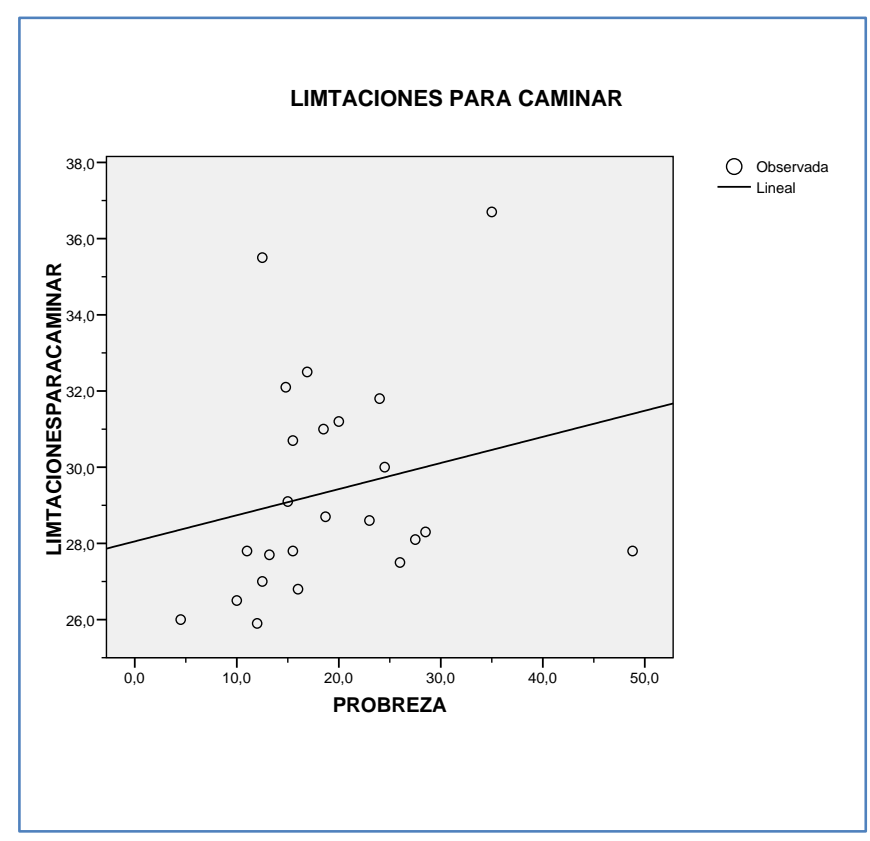

Fuente: elaborado por las autoras

Figura 1. Correlación lineal entre pobreza extrema y limitaciones para caminar en 24 departamentos de Colombia

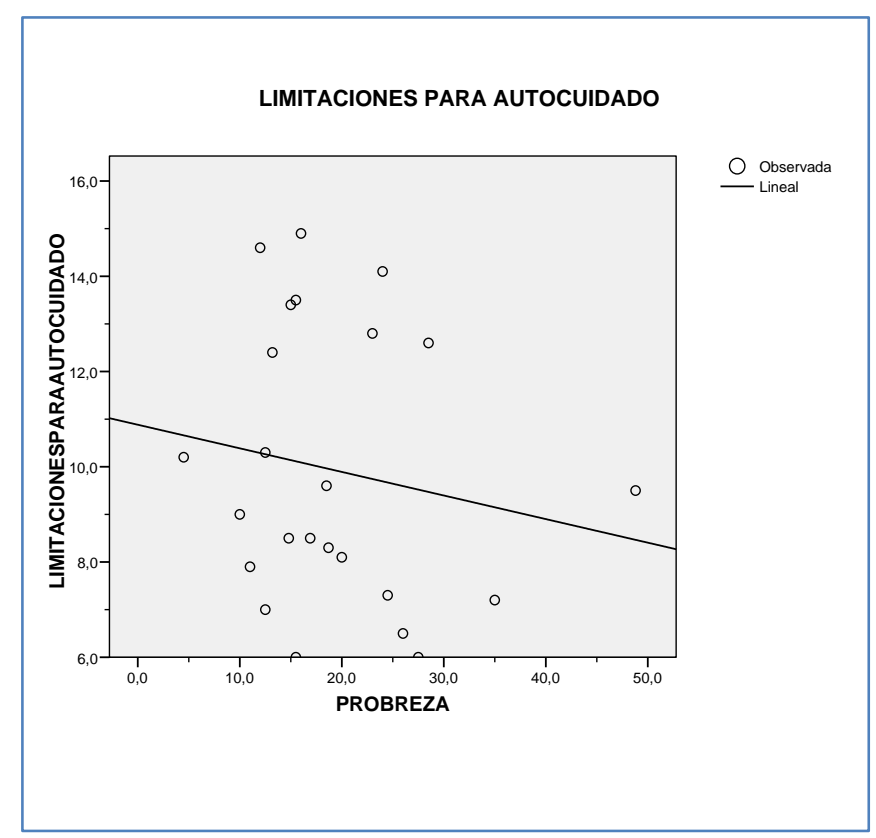

Fuente: elaborado por las autoras

Figura 2. Correlación lineal entre pobreza extrema y limitaciones para autocuidado en 24 departamentos de Colombia

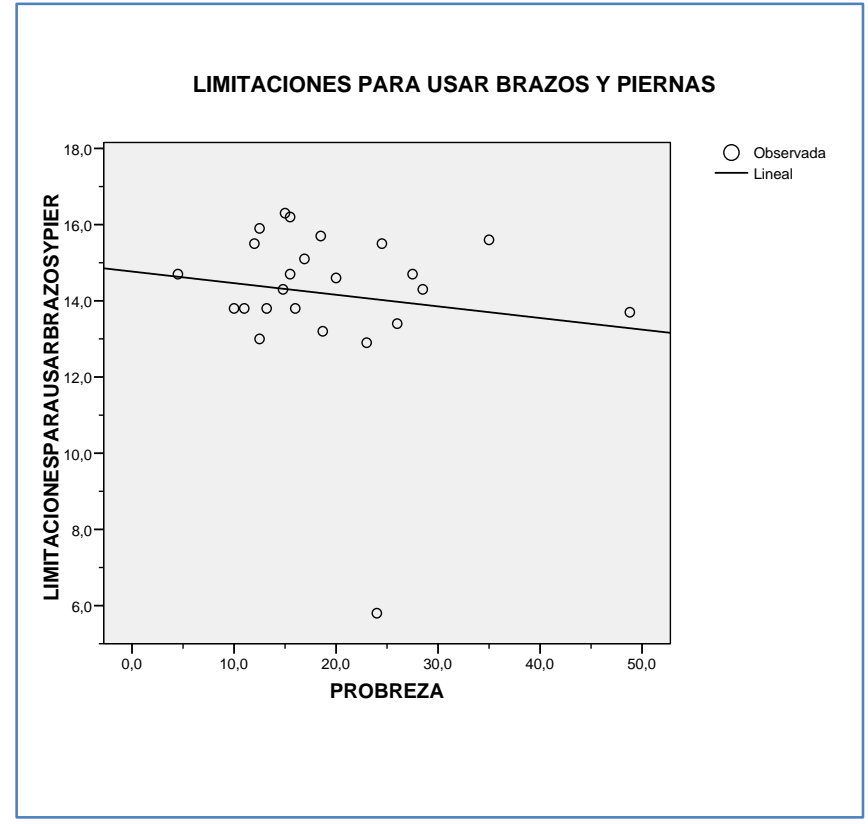

Fuente: elaborado por las autoras

Figura 3. Correlación lineal entre pobreza extrema y limitaciones para usar brazos y piernas en 24 departamentos de Colombia

\section{Discusión}

En este estudio, se evidencia que cuando la pobreza aumenta, las limitaciones para caminar son mayores. Estos coeficientes de correlación (r) indican que la pobreza influye en la situación de discapacidad de las personas en forma acorde con la actividad que se analice. En el caso de la actividad caminar, la pobreza contribuye en un $23 \%$ a que los individuos presenten mayores barreras para deambular y, por tanto, para desplazarse en su entorno.

Estos resultados permiten deducir que la pobreza es un factor contextual influyente en la discapacidad, hallazgo consistente con lo expresado por la OMS, en cuanto a que la discapacidad y la pobreza son dos variables muy relacionadas en la mayoría de los países en vías de desarrollo (13). Las posibles razones para esta correlación positiva son las pocas oportunidades que tiene una persona, en situación de discapacidad y con pocos ingresos, para acceder a servicios de salud de calidad y a mejores oportunidades educativas y laborales, todo lo cual disminuye la capacidad de generar ingresos (14).

Igualmente, los factores de riesgos para enfermedades crónicas, que producen mayores índices de discapacidad, están asociados a niveles 
socioeconómicos bajos. Es así como bajos niveles de actividad física, inadecuados hábitos alimenticios y alto consumo de alcohol y tabaco, que son factores de riesgos que llevan a discapacidad, tienen mayor ocurrencia en las poblaciones menos favorecidas (3). Por ejemplo, en Uganda, las personas en situación de discapacidad tienen un $40 \%$ de mayor probabilidad de ser pobres y los niños provenientes de hogares con familiares discapacitados cuentan con menos posibilidades de asistir a la escuela (7).

Liao (15) manifiesta que el estatus socioeconómico, medido por el nivel de educación y el ingreso, se relaciona con el aumento de mortalidad, morbilidad y discapacidad en los últimos años de vida de una persona. Al mismo tiempo, las limitaciones funcionales de una persona están fuertemente asociadas al bajo nivel de educación y aun bajo nivel de rendimiento escolar, incluso, según el estudio de Parahyba (16), esta relación es mayor en mujeres que viven en zonas rurales.

El modelo de pobreza y discapacidad de Lusting y Strauser (17) explica los factores que aumentan el riesgo de discapacidad en los individuos pobres: desvalorización del rol social, factores ambientales de riesgo, disminución del sentido de coherencia e influencias negativas del grupo social.

Se concluye que el porcentaje de pobreza extrema aumenta principalmente en proporción a las limitaciones para caminar. En específico, para el caso de Colombia, se observa que, por ejemplo, Bogotá presenta un nivel de pobreza extrema de $4 \%$ y un $26 \%$ de limitaciones para caminar; del mismo modo, el departamento de Bolívar, que tiene $13 \%$ de pobreza extrema, refleja un $26.7 \%$ de limitaciones para caminar.

Posiblemente, debido a la naturaleza multicausal de la situación de discapacidad de una persona, los coeficientes de correlación, aunque positivos, presentaron valores bajos. Por lo anterior, podría inferirse que otros factores contextuales como barreras en el ambiente físico, y factores personales como el género, edad, motivaciones, creencias, entre otras, también influyen en que un grupo de personas, en situación de discapacidad y pobres, presenten mayores limitaciones para caminar, para realizar actividades de autocuidado y para usar sus brazos y piernas.

La discapacidad depende de una serie de determinantes biológicos, psicológicos y sociales, aunque en Colombia no están claras las condiciones de vida que producen y exacerban la situación de discapacidad (18). La limitación para caminar, principal restricción relacionada con la pobreza extrema, podría ser el resultado de barreras en la residencia, en las vías públicas, en el transporte, en los centros comerciales. De hecho, los escenarios son poco accesibles para las personas que se desplazan con ayudas externas, como silla de ruedas o muletas, y si son pobres, se incrementa la exclusión de oportunidades de empleo e ingresos.

En síntesis, los resultados de este estudio permiten formular la siguiente hipótesis: la pobreza extrema es un factor causal de las limitaciones y restricciones de una persona con deficiencias físicas o mentales. De todos modos, es necesario diseñar otros tipos de estudio para estimar la asociación entre las variables estudiadas en el nivel individual. Una limitación del presente estudio es, justamente, que los resultados no se pueden ampliar de manera precisa a los individuos debido a la naturaleza grupal de los datos.

Se recomienda diseñar estudios analíticos que permitan establecer las relaciones causales entre discapacidad y pobreza en el nivel individual. También se sugiere proponer estrategias de promoción de la salud y prevención de la discapacidad en las comunidades menos favorecidas, así como fomentar hábitos de vida saludable encaminados a reducir los riesgos y mejorar el proceso de salud de la población y de los individuos. Por último, es importante el trabajo intersectorial del poder público y el privado en la formulación de políticas de salud que favorezcan a la población de menos recursos.

\section{Referencias}

1. Organización Mundial de la Salud. CIF: Clasificación Internacional del Funcionamiento, de la Discapacidad y de la Salud. OMS/OPS; 2001.

2. Jiménez BM, González DP, Martín MJ. La Clasificación Internacional del Funcionamiento de la Discapacidad y de la Salud (CIF) 2001. Rev Esp Salud Pública. 2002; 76 (4): 271-279.

3. Minkler M, Fuller TE, Guralnik J. Gradient of Disability across the Socioeconomic Spectrum in the United States. N Engl J Med. 2006; 355: 695703.

Cienc. innov. salud. Junio 2013; 1 (1): 11 - 17. Universidad Simón Bolívar (Col). ISSN: 2344-8636 
4. Consejo Nacional de Política Económica y Social, Departamento Nacional de Planeación. Red de protección social contra la extrema pobreza. Documento CONPES Social. Bogotá (D.C): 2006.

5. Hernández Y, Hernández I. Una aproximación a los Costos Indirectos de la Discapacidad en Colombia. Rev Salud Pública. 2005; 7: 130-144.

6. Mitra, S. Discapacidad es el lado oculto de la pobreza Africana. Disability World (serial online) $2004 \quad$ Enero-Marzo; $22 . \quad$ Disponible en:http://www.disabilityworld.org/01-

03_04/spanish/noticias/africa.shtml

7. Hoogeveen J. Measuring Welfare for Small but Vulnerable Groups: Poverty and Disability in Uganda. Journal of African economies. 2005; 14 (4): 603-631.

8. Beckles, B. Pobreza y discapacidad. Abogando para eliminar la exclusión social. Experiencias en Asia y América Latina y El Caribe. Coordinación del Departamento de Desarrollo Sostenible. BID. 2004.

9. Basta NE, Matthews FE, Chatfield MD, Brayne C.Community-level socio-economic status and cognitive and functional impairment in the older population. European Journal of Public Health. 2006; 18(1):48-54.

10. Cruz VI, HernándezJJ. Magnitud de la discapacidad en Colombia: una aproximación a sus determinantes. Rev. Cienc. Salud. 2008; 6(3): 23-35.

11. Departamento Administrativo Nacional de Estadística DANE. Base de Datos del Registro de Localización y Caracterización de Personas con Discapacidad. Bogotá (DC): Dirección de Censos y Demografía; 2005-2006.

12. Consejo Nacional de Política Económica y Social, Departamento Nacional de Planeación. Política Pública Nacional de Discapacidad. Documento CONPES Social. Bogotá (D.C): 2004.

13. World Health Organization. CBR a strategy for rehabilitation, equalization of opportunities, poverty reduction and social inclusion of people with disabilities: joint position paper. Geneva: 2004.
14. Urquieta SJ, Figueroa JL, Hernández PB. El gasto en salud relacionado con la condición de discapacidad. Un análisis en población pobre de México. Salud Pública de México. 2008; 50(2):136-146.

15. Liao, MD, McGee DL, Kaufmnan JS, Cao G, Cooper RS. Socioeconomic Status and Morbidity in the Last Years of Life. Am J Public Health. 1999; 89(4):569-572.

16. Parahyba MI, Veras R, Melzer D. Incapacidad e funcional entre as mulheres idosas no Brasil. Rev Saude Publica. 2005; 39(3): 383-91.

17. Lustig DC, Strauser DR. Causal relationships between poverty and disability. Rehabil Couns Bul Summer. 2007; 50(4):194-202.

18. Cruz VI, Hernández JJ. Magnitud de la discapacidad en Colombia: una aproximación a sus determinantes. Rev Cienc Salud. 2008; 6(3): 23-35. 